\title{
$\bullet$ A Succinct Analysis for Deep Learning in Deep Vision and its Applications
}

\section{IJCRR}

Section: Healthcare

ISI Impact Factor

(2019-20): 1.628

IC Value (2019): 90.81

$\operatorname{SJIF}(2020)=7.893$

\section{Preethi ${ }^{1}$, W. Jai Singh ${ }^{2}$}

'Department of Data Science, CHRIST (Deemed to be University), Pune, Lavasa India; ${ }^{2}$ Department of Computer Applications, Kumaraguru Col-

lege of Technology, Coimbatore, Tamilnadu, India.

\section{ABSTRACT}

Introduction: Deep learning methodologies can achieve forefront results on testing deep vision issues, for instance, picture portrayal, an object area, face affirmation, Natural Language Processing, Visual Data Processing and online life examination. ConvNet, Stochastic Hopfield network with hidden units, generative graphical model and sort of artificial neural network castoff to absorb competent information coding in an unproven way are deep learning plans used in deep vision issues.

Objection: This paper gives a succinct survey of without a doubt the most critical Deep learning structures. Deep vision assignments, for instance, object revelation, face affirmation, Natural Language Processing, Visual Data Processing, web-based life examination and their utilization of this task are discussed with a short record of the historic structure, central focuses and impairments. Future headings in arranging Deep learning structures for Deep vision issues and the troubles included are analysed.

Method: This paper consists of surveys. In Section two, Deep Learning Approaches and Changes are audited. In section three, we tend to portray the uses of Applications of deep learning in deep vision. In Section four, Deep learning challenges and directions are mentioned. At long last, Section five completes the paper with an outline of the results.

Results and Conclusion: Though deep learning can recall a huge proportion of data and info, it's feeble cognitive and perception of the data makes it a disclosure answer for certain applications. Deep learning despite everything encounters issues in showing various erratic facts modalities at the equal period. Multimodal profound learning is an extra notable heading in progressing deep learning research.

Key Words: ConvNet, Stochastic Hopfield network, Generative graphical model, Social media analysis, Data processing, Deep Learning

\section{INTRODUCTION}

Deep vision is an integrative intelligent arena that oversees in what way deep tin be ended to build raised equal appreciation since deep pictures or chronicles. Since the viewpoint of building, it attempts to deep endeavours that the hominid graphic structure tin do. ${ }^{1}$ Deep learning grants machine models of various taking care of layers to be told and address knowledge with various degrees of reflection addressing however the neural structure sees and grasps multimodal data, as needs are unquestionably getting included structures of colossal extension data. What perceived deep vision from the basic field of cutting edge picture getting ready around then remained a yearning toward isolate three-D structure after pictures by the area of accomplishing complete act thoughtful. Training during the 1970s moulded the initial basics aimed at countless the deep vision figuring that happen nowadays, as well as withdrawal of limits as of pictures, naming of appearances, non-polyhedral and polyhedral illustrating, the depiction of articles as inter associates of humbler constructions, visual stream, and development approximation. ${ }^{2}$ The desire to form a structure that reproduces the human brain drove the elemental progression of neural frameworks. In 1943, McCulloch and Pitts ${ }^{5}$ endeavoured toward realizing however the neural structure might create uncommonly complicated models by victimization organized developed cells, known as neurons. The McCulloch and Pitts prototypical of a vegetative cell, known as an MCP prototypical, has created a vital duty toward the advance of pretending neural frameworks.

\section{Corresponding Author:}

Preethi N, Department of Data Science, CHRIST (Deemed to be University), Pune, Lavasa India.

Email: preethinanjundan@gmail.com

ISSN: 2231-2196 (Print) ISSN: 0975-5241 (Online)
Received: 07.09 .2020
Revised: 09.11 .2020
Accepted: 18.12 .2020
Published: 20.03 .2021 
Deep learning has drove fantastic strolls in briefing of computer vision complications, let's say, entity identification, movement following, activity acknowledgment, human skill estimation, and linguistics division. ${ }^{3-7}$ During this arrangement, we'll minimal rustically evaluation the core progressions in deep learning models besides figuring for computer vision bids during the extraordinary circumstance, Three of the foremost vast sorts of deep learning prototypical with relevancy their significance in visual kind, that is, ConvNet, the "Boltzmann special" composed with Deep Belief Networks (DBNs) and Deep Boltzmann Machines (DBMs) and Stacked (Denoising) Autoencoders.

This paper consists of surveys. In Section two, Deep Learning Approaches and Changes are audited. In section three, we tend to portray the uses of Applications of deep learning in deep vision. In Section four, Deep learning challenges and directions are mentioned. At long last, Section five completes the paper with an outline of the results.

\section{DEEP LEARNING APPROACHES AND CHANGES}

\section{ConvNet}

ConvNet victimization the boner incline and accomplishing usually astounding ends up in a briefing of model affirmation tasks. ${ }^{8}$ ConvNet is spurred by the visual schemes assembled, and expressly through its prototype planned in. ${ }^{9}$ The principle machine prototype dependent unprocurable systems among neurons and logically created changes out of the copy are created in Neocognitron, which delineates that once neurons through comparative limits are suitable on areas of the past pane by completely dissimilar zones, a method of change of location in vacillation is secured.

A CNN incorporates 3 commonplace forms of neural layers, to be express, (a) ConvNet Phase, (b) combining layers, and (c) whole Merging layers. Variety of phase settles for substitute activity. Figure 1 shows a $\mathrm{CNN}$ building for a piece characteristic proof in copy mission. Each phase of a Con-

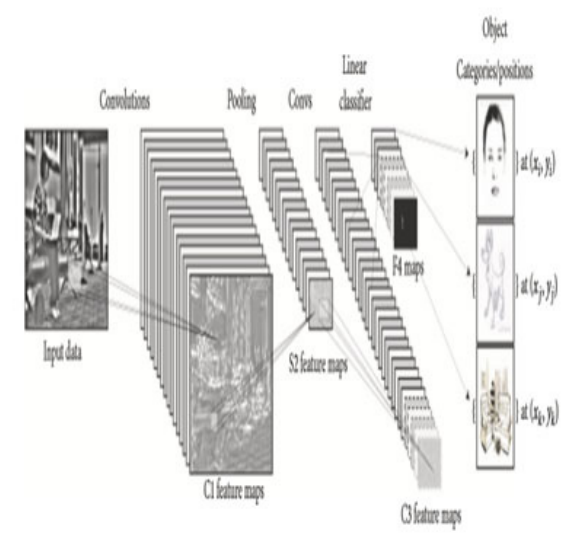

Figure 1: Instance building of a CNN for a Deep vision task(object location).
vNet changes info capacity to a yield capacity of somatic cell commencement, over the long-term agitative the last whole connected layers, achieving a mapping of the info to a 1D embody vector. CNN's are exceptionally productive in deep vision applications, to Illustrate, face affirmation, object revelation, driving idea in apply autonomy vehicles.

a) ConvNet Phase. ConvNet Phase, a ConvNetusesnumerous bit to rotate the entire copy similarly to the midway component maps, delivering diverse component plots. As the advantages of the complication action, a couple of task have planned it as an additional for totally associated phase through the ultimate objective of achieving speedier learning events.

b) Combining Phase: Combining Phaseremainin charge of diminishing the three-dimensional estimations of the data capacity aimed at the following difficult phase. The combining phase doesn't impact the significance estimation of the capacity. This action achieved through this lateral indicated sub examining or down testing, by way of the diminishing of scope prompts a concurrent lack of evidence. In any case, mishap may be helpfully aimed at the framework because the decline in dimensions prompts fewer figures overhead used for the future phases of the framework, also besides that kills overflow. Typical combing and max combining are the maxima generally applied procedures. Distinct academic examination of max combining and ordinary combining shows is assumed, and exhibited the most extreme combing could provoke speedier intermixing, select dominating constant structures, and upgrade hypothesis. Different various assortments of the combining phase of the composition, inspired through changed motivation sand helping indisputable necessities. $^{11}$

c) Completely Linked Layers. Following one or two complexity and combining phase, large phase intuition within the neural framework is achieved by strategies for wholly associated phase. Neurons in an exceedingly wholly associated phase take complete relationship with completely sanctionative within past phase, as per their tag recommends. In the beginning, this point forward is non-commissioned with a structured growth followed by an inclination offset. wholly associated phase ineluctably change the 2 nd article plots into a 1D embrace path. The result and path moreover may well remain an addressed advancing to selected ranging orders used for a game set up or might remain thought-about as per a path for additional method. ${ }^{12}$

ConvNet structure uses three strong attention: (a) area responsive range, (b) fixed burdens, and (c) Structural subsample. Taking into account close by responsive range, every component in a convolutional layer gets ideas of neighbouring components having a spot with the last layer. Thusly neurons are fit for removing simple visual features, for instance, boundaries or joints. The above-mentioned selections 
are formerly joined through the following complex phases acknowledge complex solicitation structures. Plus, straight forward part locators, that are helpful on a touch of a picture, are presumptively attending to be vital over the complete figure is ample through the chance of joint burdens. The joint burdens goal is a good deal of items toward its vague burdens. Decidedly, the elements of a convolutional layer sifted through the sphere. Entireelementsset up supply a comparable game plan of burdens. Hence, every plane is in danger of building a selected part. The yields of the sphere are known as structural plots. Every convolutional layer involves one or two planes, thus varied phase maps are created in every region. throughout the advancement on the part plot, these complete figures are checked through elements that are taken care of at staring at regions within the phrase maps. This improvement is like a convolution action, trailed by an extra substance tendency period and sigmoid edge:

$$
\mathbf{y}^{(d)}=\sigma\left(\mathbf{W y}^{(d-1)}+\mathbf{b}\right),
$$

Here, $d$ states the importance of the convolutional layer, the weight cross-section is denoted by $\mathrm{W}$, and the inclination term is denoted by b. Completely associated neural frameworks, burden lattice is filled, that is, interfaces all commitment to the respective element through totally unlike burdens. For ConvNet, $\mathrm{W}$ is the burden system which is too little visible of the chance of tied burdens.

$$
\left[\begin{array}{cccc}
\mathbf{w} & 0 & \cdots & 0 \\
0 & \mathbf{w} & \cdots & 0 \\
\vdots & \cdots & \ddots & \vdots \\
0 & \cdots & 0 & \mathbf{w}
\end{array}\right],
$$

Here $\mathrm{w}$ is matrices taking comparative estimations with the units' open fields. victimisation associate inadequate weight matrix diminishes the quantity of the framework's tunable parameters and thus grows its theory limit. increasing $\mathrm{W}$ with layer inputs takes once convolving the commitment with $\mathrm{w}$, which might be seen as a trainable channel. If the commitment to $d-1$ convolutional layer is of estimation $N \times N$ and therefore the responsive field of units at a particular plane of convolutional layer $d$ is of estimation $m \times m$, by then the created element guide is going to be a structure of estimations $(N-m+1) \times(N-m+1)$. Precisely, the phase of feature plot at $(i, j)$ tract is going to be

$$
\mathbf{y}_{i j}^{(d)}=\sigma\left(x_{i j}^{(d)}+b\right)
$$

With

$$
x_{i j}^{(d)}=\sum_{\alpha=0}^{m-1} \sum_{b=0}^{m-1} w_{\alpha b} \mathbf{y}_{(i+\alpha)(j+b)}^{(d-1)},
$$

Here the scalar is b. Using (2) and (3) consecutively for entirely $(i, j)$ spots of knowledge, the half plot for the relating plane is made.

The difficulties which will develop by preparing of CNNs needs to fix with the tremendous variety of strictures which has got acknowledged, that can incite the effort by overfitting. to the present finish, frameworks, maybe, random pooling, dropout, and information development are planned. additionally, CNNs are as typically as attainable assumed to pre-processing, which is, a technique that instates the framework by pre-processing parameters as hostile without aim set ones. Pretraining will enliven the educational technique and update the hypothesis limit of the framework. All things thought-about, CNNs were looked as if it would primarily trump commonplace AI methods is a very wide extent of Deep vision and model affirmation errands, ${ }^{13}$ samples are given in Section3. Its splendid show got alongside the relative simplicity in preparing are the essential reasons that specify the unfathomable arrive of their predominance in the course of the most recent number of years.

\section{Generative graphical model and Stochastic Hopfield network with hidden units}

Deep Belief Networks and Deep Boltzmann Machines are Deep learning models that belong to the "Boltzmann family," as they utilize the Restricted Boltzmann Machine (RBM) as a knowledge module. The generative stochastic artificial neural network is also called the Restricted Boltzmann Machine (RBM). DBNs have an objectiveless relationship by two layers that structure an RBM and guiding relationship with the lesser layers. The generative graphical model has directionless association among full layers of the framework. An apt representation of DBNs and DBMS can be initiate in (Figure 2). In going with subclasses, we will depict the major characteristics of DBNs and DBMs, in the wake of presenting their fundamental structure hinder, the RBM.

\section{Generative graphical model}

Deep Belief Networks (DBNs) are probabilistic generative models that provide a probability transport ended perceptible information and also names. That is shaped by loading RBMs and setting them up in a covetous way, which are planned in. ${ }^{14}$ A DBN from the start uses a helpful layer-by-layer energetic knowledge procedure to gift many frameworks, and, within the facet project, adjusts all plenty in conjunction with the right yields. DBNs are graphical models that add up the way to evacuate a big dynamic depiction of the readiness 
information. They model the be a part of $t$ spread between watched vector $\mathrm{x}$ and also the $l$ lined layers $\mathrm{h} k$ as follows:

$$
P\left(\mathbf{x}, \mathbf{h}^{1}, \ldots, \mathbf{h}^{l}\right)=\left(\prod_{k=0}^{l-2} P\left(\mathbf{h}^{k} \mid \mathbf{h}^{k+1}\right)\right) P\left(\mathbf{h}^{l-1}, \mathbf{h}^{l}\right),
$$

where $\mathrm{x}=\mathrm{h} 0, P(\mathrm{~h} k \mid \mathrm{h} k+1)$ is Associate in Nursing unforeseen alternative aimed at the perceptible components at level $k$ adjusted arranged the lined components of the RBM at equal $k+1$, and $P(\mathrm{~h} l-1 \mid \mathrm{h} l)$ remains that the taken for granted - lined joint unfold within the top-ranking RBM.
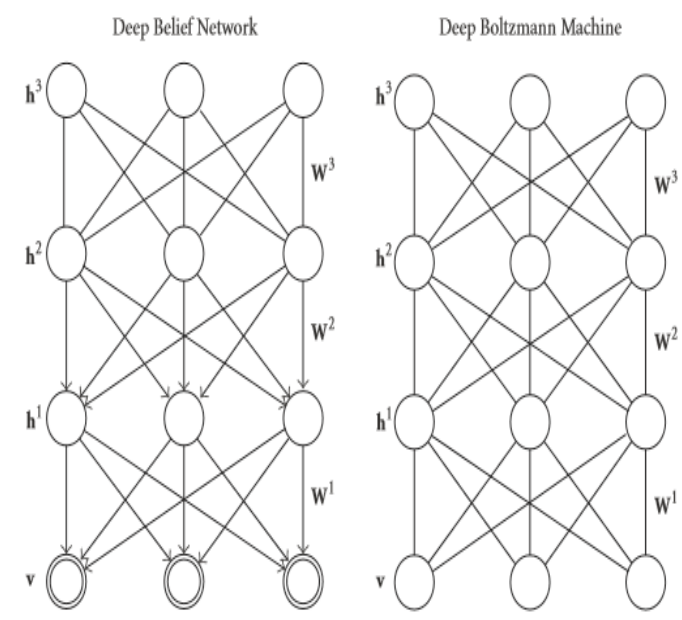

Figure 2: Deep Belief Network(DBN)and Deep Boltzmann Machine (DBM).

The top two layers of a DBN structure a directionless outline $\mathrm{n}$ and the remainder of the layers structure a conviction facilitate by composed, top-down affiliations. In a DBM, all affiliations are directionless.

The standard of $m$ voracious layer-wise freelance coming up with is functional to DBNs by RBMs because the structure frustrates for every layer. ${ }^{15} \mathrm{~A}$ summary portrayal of the system is as follows:

(1) Train the chief layer as an RBM which models the rough info $\mathrm{x}=\mathrm{h} 0$ as its perceptible layer.

(2) Practice that 1st layer to secure an outline of the info which may be used as data for the following layer. 2 typical game plans exist. This depiction is picked just like the mean authorization $(\mathrm{h} 1=1 \mid \mathrm{h} 0)$ or trial of (h1|h0).

(3) Train the second layer as an RBM, which modified information (tests or mean commencement) as coming up with examples(for the conspicuous layer of that RBM).

(4) Restate steps ((2)and(3)) for the right variety of layers, whenever multiplying upward either test or mean characteristics

(5) Fine-tune all the limits of this vital structure with relevancy a middle person for the DBN log-likelihood, or concerning a regulated coming up with live (consequent to adding further learning device to vary over the perceptive depiction into oversaw gauges, e.g., an instantaneous classifier).

There are 2 essential inclinations within the above-depicted unsatiable learning methodology of the DBNs. ${ }^{16}$ First, it handles the trial of acceptable assurance of parameters, that once during a whereas will incite poor within reach optima, as desires are guaranteeing that the framework is befittingly conferred. Second, there's no essential for checked information since the system is freelance. Regardless, DBNs are in a like manner full of totally different deficiencies, parenthetically, the procedure value connected with putting in a DBN and also the manner that the strategies towards any improvement of the framework dependent on most outrageous chance preparing gauge are cloudy. ${ }^{15}$ to boot, a very important obstruction of DBNs is that they don't speak to the 2D structure of an information image, which can primarily impact the show and connection in deep vision and sight and sound examination problems. Regardless, a later assortment of the DBN, the CDBN is a generative graphical model. ${ }^{17}$ uses the three-dimensional info of neighbouring pixels by presenting convolutional RBMs, afterwards creating a change invariant generative model that with success scales with relevancy high dimensional photos.

\section{APPLICATIONS OF DEEP LEARNING IN DEEP VISION}

These days, employments of Deep learning join anyway are not obliged to NLP (e.g., sentence gathering, translation, etc.), visual data dealing with (e.g., Deep vision, blended media data assessment, etc.), talk and sound getting ready (e.g., overhaul, affirmation, etc.), relational association examination, and restorative administrations. This portion offers nuances to the unrelated techniques used for the respective application.

\section{Natural Language Processing}

NLP is a movement of estimations and frameworks that essentially based on demonstrating deep to fathom the human language. NLP endeavours fuse report portrayal, understanding, revise recognizing confirmation, content closeness, summary, and question answering. NLP progression is attempting a direct result of the multifaceted nature and unsure building of the human language. Furthermore, ordinary language is significantly setting express, where severe ramifications change subject to the kind of words, joke, and region distinction. Significant learning systems have starting 
late had the choice to show a couple of compelling undertakings in attaining high precision in NLP errands.

Most NLP models follow a practically identical pre-planning step: (1) the information content is isolated into words by tokenization and a while later (2) these words are rehashed as vectors or $\mathrm{n}$-grams. Addressing words in a low estimation is fundamental to make a precise perspective on similarities and differentiation among numerous words. The test shows up when there is essential to pick the length of words limited in each n-gram. This strategy is setting express and needs previous region data. A part of the outstandingly noteworthy systems in appreciating the most prominent NLP assignments are presented underneath.

\section{Sentiment Analysis}

This bit of NLP supervises looking at a book and organizing the propensity or evaluation of the author. Maximum datasets for end assessment are separate as either confident or undesirable, and reasonable enunciations are expelled by bias demand philosophies. The single commended model is the Standford Sentiment Treebank (SST),${ }^{18}$ a dataset of film surveys set apart into five classes (going from negative to unfathomably positive). Near to the preface to SST, Socher et al. ${ }^{18}$ propose a Recursive Neural Tensor Network (RNTN) that usages word vectors and parses a tree to address an enunciation, getting the coordinated efforts among the parts with a tensor-based strategy work. This recursive methodology is perfect concerning sentence-level depiction since the emphasis reliably shows a tree-like structure.

$\mathrm{Kim}^{19}$ improves the correctness for SST by next a substitute technique. Notwithstanding the way that CNN models were first made considering picture insistence and strategy, their execution in NLP has displayed to be a triumph, accomplishing brilliant outcomes. Kim grants a direct CNN model utilizing one convolution layer on masterminded word2vec vectors in a BoW structure. The representations were spared sensibly crucial with relatively few hyperparameters for change. By a mix of low tuning and pre-trained task-express parameters, they understand how to accomplish high precision on two or three standards. Online life is a prominent wellspring of information while dissecting notions.

\section{Machine Translation}

Deep learning has accepted a noteworthy activity in the updates of customary customized translation systems. Cho et al. ${ }^{20}$ presented a new RNN-based encoding and unwinding configuration to set up the words in a Neural Machine Translation (NMT). The RNN Encoder-Decoder framework practices two RNNs: one plot a data progression into fixedlength vectors, however, the other RNN translates the vector into the goal pictures. The problem with the RNN EncoderDecoder is the introduction fall as the data course of action of pictures extends. Bahdanau et al. ${ }^{16}$ address this question by introducing a dynamic-length vector and by together knowledge the alter and disentangle techniques. Their approach is to play out a matched mission to scan for syntactic structures that are commonly judicious for understanding. In any case, the starting late planned translation schemes are known to be computationally classy and incompetent in dealing with sentences covering unprecedented words.

\section{Paraphrase Identification}

Rework distinguishing proof is the path toward separating two sentences and foreseeing how equivalent they rely upon their essential covered semantics. A key part that is useful for a couple of NLP occupations, for instance, copyright encroachment acknowledgement, answers to questions, setting area, abstract, and region recognizing evidence. Socher et al. ${ }^{18}$ suggest the usage of spreading out Recursive Autoencoders (RAEs) to amount the resemblance of two sentences. Using syntactic trees to progress the part space, they measure both word-and articulation level comparable qualities. Notwithstanding the way that it is on a very basic level equivalent to RvNN, RAE is useful in the independent request. Not in the slightest degree like RvNN, RAE registers a generation screw up in its place of a controlled score during the meeting of two vectors into a compositional vector. This article furthermore introduced a dynamic pooling layer that can consider and bunch two sentences of unlike sizes as either a translation or not.

\section{Visual Data Processing}

Deep learning techniques have developed the central bits of numerous front line intelligent media systems and Deep vision. ${ }^{21}$ Even more unequivocally, CNNs have exhibited basic results in different genuine endeavours, including picture taking care of, object acknowledgement, and video getting ready. This zone discusses more bits of knowledge concerning the most recent significant learning structures and estimations planned over the late years for visual data taking care of.

\section{Image Classification}

In 1998, LeCun et al. prevailing the essential type of LeNet-5 [43]. LeNet-5 is a standard CNN that joins two convolutional layers close by a subselection layer ultimately getting done through a complete relationship in the previous layer. Despite the way that, since the mid-2000s, LeNet-5 and other CNN strategies were hugely used in dissimilar issues, counting the division, area, and portrayal of pictures, they were nearly rejected by data mining and AI study get-togethers. Over a multi decade later, the CNN figuring has started its thriving in Deep vision systems. Exactly, AlexNet ${ }^{22}$ is seen as the first CNN prototype that significantly enhanced the image portrayal consequences on an amazingly colossal dataset 
(e.g., ImageNet). It was the victor of the ILSVRC 2012 and improved the best results from the prior years by for all intents and purposes $10 \%$ concerning the best five test bumble. To recover the effectiveness and the rapidity of setting up, a GPU execution of the CNN is used in this framework. Data increment and dropout methods are furthermore used to altogether lessen the overfitting issue.

\section{Object Detection and Semantic Segmentation}

Deep learning methodology accepts a noteworthy activity in the movement of article distinguishing proof starting late. Before that, the best article acknowledgement execution began from complex structures with a couple of low-level structures (e.g., SIFT, HOG, etc.) and huge level settings. In any case, with the methodology of new significant learning frameworks, object ID has in like manner showed up at another period of progress. These advances are driven by compelling methodologies, for instance, zone recommendation and section-based CNN (R-CNN). ${ }^{23} \mathrm{R}-\mathrm{CNN}$ defeats any obstruction among the article area and picture game plan by presenting section grounded thing repression strategies using deep frameworks. Besides, the move to learn and relating on a colossal dataset (e.g., ImageNet) is applied since the little thing acknowledgement datasets (e.g., PASCAL [46]) fuse lacking named data to set up a gigantic CNN sort out. In any case, in R-CNN, the readiness computational time and memory are over the top costly, particularly on novel ultrasignificant frameworks (e.g., VGGNet).

\section{Video Processing}

Video examination has pulled in broad thought in the deep vision organize and is measured as a troublesome task since it consolidates mutually spatial and brief data. In an early slog, colossal degree YouTube accounts containing 487 game classes are used to set up a CNN model. ${ }^{24}$ The model fuses a multiresolution designing that employs the close-by development data in accounts and joins setting stream (for low-objectives picture illustrating) and fovea stream (for significant standards picture dealing with) modules to arrange chronicles. An occasion acknowledgement from game chronicles using significant learning is presented. ${ }^{25}$ In that work, both spatial and brief data are determined using CNNs and feature mix through standardized Autoencoders. Starting late, another framework called Recurrent Convolution Networks (RCNs) was introduced for video dealing. It smears $\mathrm{CNNs}$ on video traces for pictorial comprehension and a short time later deals with the housings to RNNs for exploring transient information in accounts.

\section{Social Media Analysis}

\section{Social Web Analysis}

The notoriety of various relational associations like Facebook and Twitter has engaged customers to part a ton of data with their photographs, considerations, and sentiments. Because of the way that significant knowledge has revealed hopeful execution on visual data and NLP, unmistakable significant learning methods have been grasped for relational association examination, including semantic evaluation, interface estimate, and crisis response. ${ }^{26,27}$ The semantic appraisal is a huge field in casual association assessment, which means to help machines with understanding the semantic significance of posts in relational associations. Though a collection of techniques have been planned to separate works in NLP, these strategies might disregard to address a couple of standard difficulties in relational association assessment, for instance, spelling botches, abbreviated structures, unprecedented characters, and easy-going vernaculars. ${ }^{28}$

Twitter can be considered as the most routinely used wellspring of appraisal request for relational association examination. Generally speaking, feeling examination hopes to choose the mien of analysts. Consequently, SemEval has given a standard dataset reliant on Twitter and run the assessment game plan task since 2013. Another practically identical model is Amazon, which ongoing as an online book shop and is as of now the world's greatest online retailer. With an abundance of acquirement trades, a colossal proportion of evaluations are made by the clients, making the Amazon dataset a remarkable hotspot for tremendous extension estimation plan. ${ }^{29}$

\section{Information Retrieval}

Deep adapting incredibly influences information recuperation. Deep Structured Semantic Modelling (DSSM) is planned for text recuperation and web search, ${ }^{30}$ where the dormant semantic examination is driven by a DNN and the inquiries nearby the explore data are used to choose the eventual outcomes of the recuperation. The encoded requests and explore data are mapped into $30 \mathrm{k}$-estimation by term hashing and a 128-estimation feature space is delivered by the multilayer nonlinear plans. The proposed DNN is set up to interface the offered inquiries to their semantic criticalness with the help of the explore data. Regardless, this proposed classical treats each term autonomously and disregards the relationship among the terms.

\section{DEEP LEARNING CHALLENGES AND DIREC- TIONS}

With the extraordinary progression in profound learning and its assessment scenes existence at the centre of attention, profound learning has expanded excellent power in talk, language, and visual revelation structures. In any case, a couple of spaces are still faultless by DNNs owing to either their troublesome countryside or the nonattendance of data openness for the overall populace. This makes important possibilities and productive ground for compensating upcoming 
study streets. The most significant future AI issues won't have sufficient getting ready tests with names. ${ }^{31}$ Beside the zettabytes of starting at now open data, petabytes of data are incorporated every day. This exponential improvement is gathering data that can never be named by human aid. The current estimation is pleasing to coordinated learning, by and large under the immediately open imprints and the little sizes of current datasets. Regardless, with the brisk augmentations in the scope and unpredictability of information, independent learning will be the predominant course of action later on. Current profound learning models will in like manner need to conform to the rising issues, for instance, data sparsity, missing data, and disordered data to get the approached in forever discernments as opposed to getting ready.

Another achievement challenge looked at by profound learning techniques is the decline of dimensionality without losing fundamental information required for request. In clinical applications like danger RNA sequencing examination, generally, the amount of tests in each imprint is far not the number of features. In current profound learning models, this causes outrageous overfitting issues and limits the fitting course of action of lacking cases. Current profound learning structures require broad proportions of computational advantages for a push toward the front line presentations. One system attempts to vanquish this test by using store preparing. Added different is to custom the slow systems that misuse medium and colossal datasets on separated getting ready. In rhythmic movement years, various researchers have moved fixation to develop equivalent and versatile profound learning frameworks.

\section{CONCLUSION}

Deep learning, another and fervently discussed the issue in $\mathrm{AI}$, can be described as a course of layers acting nonlinear getting ready to get comfortable with various degrees of data depictions. This article contemplates the top tier counts and methods in deep learning. A couple of disclosures of this article and likely future work are dense underneath:

- Thoughdeep learning can recall a huge proportion of data and info, it feels cognitive and perception of the data makes it a disclosure answer for certain applications. The interpretability of deep learning should be inspected later on.

- Deep learning despite everything encounters issues in showing various erratic facts modalities at an equal period. Multimodal profound learning is an extra notable heading in progressing deep learning research.

- Disparate human personalities, deep adapting needs wide datasets (unmistakably named data) for coming down the machine and anticipating the inconspicuous information. This issue turns out to be all the more overwhelming when the existing datasets are pretty much nothing (e.g., social protection data) or when the data ought to be arranged ceaselessly. One-shot learning and zero-shot learning have been packed in the continuous hardly any years to help this issue.

- In disdain of all the profound learning types of progress starting late, various applications are up 'til now flawless by profound learning or are first and foremost times of using the profound learning systems (e.g., disaster information the load up, cash, or clinical data assessment).

\section{REFERENCES}

1. Ballard DH, Brown CM. Deep Vision. Prentice Hall 1982. ISBN 978-0-13-165316-0.

2. Richard Szeliski (30 September 2010). Deep Vision: Algorithms and Applications. Springer Science \& Business Media. pp. 1016. ISBN 978-1-84882-935-0.

3. Ouyang W, Zeng X, Wang X. DeepID-Net: Object Detection with Deformable Part Based Convolutional Neural Networks. IEEE Transactions on Pattern Analysis and Machine Intelligence $2017 ; 39(7): 1320-1334$.

4. Doulamis N, Voulodimos A. "FAST-MDL: Fast Adaptive SupervisedTrainingofmulti-layereddeeplearningmodelsfor consistent object tracking and classification," in Proceedings of the 2016 IEEE International Conference on Imaging Systems and Techniques 2016 pp.318-323.

5. Lin L, Wang K, Zuo W, Wang M, Luo J, Zhang L. Adeep structured model with radius-margin bound for 3D human activity recognition. International Journal of Deep Vision, 2016;118(2):256-273.

6. Toshev A, Szegedy C. DeepPose:Humanposeestimation via deep neural networks. Proceedings of the 27th IEEE Conference on Deep Vision and Pattern Recognition 2014:pp.1653-1660, USA,June2014.

7. Noh H, Hong S, Han B. Learning deconvolution network for semantic segmentation. Proceedings of the 15th IEEE International Conference on Deep Vision, ICCV 2015, pp. 1520-1528, Santiago,Chile,December2015.

8. LeCun Y, Boser B, Denkeretal JS. Back propagation applied to hand written zip code recognition. Neural Computation 1989;1(4):541-551.

9. Hubel DH, Wiesel TN. Receptive fields, binocular interaction, and functional architecture in the cat's visual cortex. J Physiol 1962; 160:106-154.

10. Oquab M, Bottou L, Laptev I, Sivic J. Is object localization for free? Weakly-supervised learning with convolutional neural networks. Proceedings of the IEEE Conference on Deep Vision and Pattern Recognition 2015, pp.685- 694.

11. Scherer D, Muller A, Behnke S. Evaluation of pooling operations in convolutional architectures for object recognition," Lecture Notes in Deep Science (including subseries Lecture Notes in Artificial Intelligence and Lecture Notes in Bioinformatics): Preface 2010;6354(3):92-101.

12. Krizhevsky A, Sutskever I, Hinton GE. Image net classification with Deep convolutional neural networks. Proceedings of the $26^{\text {th }}$ Annual Conference on Neural Information Processing Systems (NIPS '12), pp. 1097-1105, Lake Tahoe, Nev, USA, December2012.

13. Hinton GE, Salakhutdinov RR. Reducing the dimensionality of data with neural networks. Am Assoc Adv Sci 2006;313(5786):504-507. 
14. Bengio Y. Learning deep architectures for AI. Foundat Trends Mach Lear 2009;2(1):1-27.

15. Bengio Y, Courville A, Vincent P. Representation learning: a review and new perspectives. IEEE Transactions on Pattern Analysis and Machine Intelligence 2013;35(8):1798-1828.

16. Bahdanau D, Cho K, Bengio Y. Neural machine translation by jointly learning to align and translate. CoRRabs 2014;1409.0473. Retrieved from http://arxiv.org/abs/1409.0473.

17. Lee H, Grosse R, Ranganath R, Ng AY. Convolutional deep belief networks for scalable unsupervised learning of hierarchical representations. Proceedings of the $26^{\text {th }}$ Annual International Conference 2009: pp.609-616.

18. Socher R, Perelygin A, Wu JY, Chuang J, Manning CD, Ng AY, Potts C. Recursive deep models for semantic compositionality over a sentiment treebank. In Conference on Empirical Methods in Natural Language Processing. Citeseer, Association for Computational Linguistics 2013;1631-1642.

19. Yoon Kim. Convolutional neural networks for sentence classification. CoRRabs 2014:1408.5882 (2014). Retrieved from http:// arxiv.org/abs/1408.5882.79]

20. Cho K, van Merrienboer B, Gülçehre C, Bahdanau D, Bougares F, Schwenk H, Bengio Y.. Learning phrase representations using RNN encoder-decoder for statistical machine translation. In The Conference on Empirical Methods in Natural Language Processing 2014:1724-1734.

21. Ha HY, Yang Y, Pouyanfar S, Tian H, Chen SC. Correlationbased deep learning for multimedia semantic concept detection. In International Conference on Web Information Systems Engineering. Springer, 2015:473-487.

22. Krizhevsky A, Sutskever I, Hinton GE.. ImageNet classification with deep convolutional neural networks. In Advances in Neural Information Processing Systems 25, F. Pereira, C. J. C. Burges, L. Bottou, and K. Q. Weinberger (Eds.). Curran Associates, 2012: 1097-1105.

23. Girshick R, Donahue J, Darrell T, Malik J. Rich feature hierarchies for accurate object detection and semantic segmentation.
In IEEE Conference on Deep Vision and Pattern Recognition. IEEE, 2014:580-587.

24. Karpathy A, Toderici G, Shetty S, Leung T, Sukthankar R, Li FF.. Largescale video classification with convolutional neural networks. In IEEE Conference on Deep Vision and Pattern Recognition. IEEE Deep Society, 2014:1725-1732.

25. Tsagkatakis G, Jaber M, Tsakalides P. Goal!! Event detection in sports video. Electr Imaging 2017;16:15-20.

26. Donahue J, Hendricks LA, Guadarrama S, Rohrbach M, Venugopalan S, Saenko K, et al. Long-term recurrent convolutional networks for visual recognition and description. In IEEE Conference on Deep Vision and Pattern Recognition. IEEE Deep Society 2015;2625-2634.

27. Vosoughi S, Vijayaraghavan P, Roy D. Tweet2Vec: learning Tweet embeddings using character-level CNN-LSTM encoderdecoder. In The 39th International ACM SIGIR Conference on Research and Development in Information Retrieval. ACM, 2016.1041-1044.

28. Nguyen DT, Joty SR, Imran M, Sajjad H, Mitra P.. Applications of online deep learning for crisis response using social media information. CoRRabs 2016;1610.01030. Retrieved from http:// arxiv.org/abs/1610.01030.

29. Nakov P, Ritter A, Rosenthal S, Sebastiani F, Stoyanov V.. SemEval-2016 task 4: Sentiment analysis in Twitter. In The 10th InternationalWorkshop on Semantic Evaluation. Association for Deep Linguistics, 2016: 1-18.

30. Zhang X, Zhao J, LeCun Y. Character-level convolutional networks for text classification. In Advances in Neural Information Processing Systems. 2015:649-657.

31. Zhu Y, Urtasun R, Salakhutdinov R, Fidler S. SegDeepM: Exploiting segmentation and context in deep neural networks for object detection," in Proceedings of the IEEE Conference on Deep Vision and Pattern Recognition, CVPR 2015:4703-4711. 